\title{
Blokzincir Teknolojisinin Sağlık Bilgi Sistemlerinde Kullanımı
}

\author{
Mehmet Aydar $^{1 *}$, Salih Cemil Çetin ${ }^{2}$ \\ ${ }^{1}$ Ford Otosan, Kurumsal Mimari ve Teknoloji İnovasyonu Departman1, İstanbul / Türkiye (ORCID: 0000-0002-5578-758X) \\ 2 Softect, Araştırma Geliştirme Merkezi, İstanbul, Türkiye (ORCID: 0000-0003-1356-0798)
}

(İlk Geliş Tarihi 10 Mayıs 2020 ve Kabul Tarihi 17 Haziran 2020)

(DOI: 10.31590/ejosat.735052)

\begin{abstract}
ATIF/REFERENCE: Aydar, M. \& Çetin, S. C. (2020). Blokzincir Teknolojisinin Sağlık Bilgi Sistemlerinde Kullanımı. Avrupa Bilim ve Teknoloji Dergisi, (19), 533-538.

Öz

Blokzincir protokolü, şifrelenmiş bilginin zincir halindeki bloklar içerisinde dağıtık olarak saklanmasını temel alan bir konsepttir. Bu konsept, işlemleri daha güvenli ve takip edilebilir hale getirirken aynı zamanda şeffaflık sağlamaktadır. Protokol üzerinde gerçekleşen işlemler sonradan manipüle edilememekte ve kontrollü şekilde sisteme dâhil olan aktörler tarafından doğrulanabilmektedir. Bu teknolojinin son yıllarda, insanlar ve kuruluşlar arasındaki ilişkileri düzenlediği ve bu alanda yeni firsatlar sunduğu gözlemlenmektedir. Böylelikle blokzincir protokolleri, birçok sektörde kendisine yer bulabilme potansiyeline sahiptir. Bu çalışmada, sağlık sisteminde blokzincir teknolojisinden faydalanan mevcut uygulamaları ve blokzincir protokollerinin sağlık bilgi sistemlerindeki potansiyel faydalarını araştırdık.
\end{abstract}

Anahtar Kelimeler: Blokzincir protokolü, Dağıtık defter teknolojisi, Güvenilir ağlar, Sağlık bilgi sistemleri.

\section{Blockchain for health information systems}

\begin{abstract}
The blockchain protocol is a concept of protocols that relies on keeping encrypted information on distributed linked blocks. Blockchain protocols make it possible for transactions to be more efficient and traceable while providing transparency. Transactions on the protocol are immutable, and they can be verified by trusted participants in the system. The advent of blockchain technology has created new opportunities to transform how relationships between people and institutions are established and maintained, and it has proven to have disruptive use in various sectors. In this paper, we research existing studies and applications that utilize blockchain technology in health information systems, along with the potential advantages of Blockchain in health systems.
\end{abstract}

Keywords: Blockchain protocols, Distributed ledger technologies, Trusted networks, Health information systems.

\section{Giriş}

Blokzincir protokolü, bilginin birbirini refere eden bloklar içerisinde saklanmasını temel alan bir dağıtık defter teknolojisidir. Burada, bilgi sahipliği aktif şekilde kontrol edilmekte ve tutulan kayıtların birer kopyası tüm kullanıcılarda dağıtık olarak saklanmaktadır. Merkezi otoriteler yerine ademi merkeziyetçi yapıyı destekleyen bu yaklaşım, peer-to-peer ağlar üzerine konumlandırılmıştır. Peer-to-peer ağlar, geleneksel sunucu-istemci mimarisinin aksine, her istemcinin aynı zamanda bir sunucu olarak çalışması prensibine dayanır. Peer-to-peer ağları kullanan blokzincir teknolojisinde bilgi, kriptografinin de yardımıyla, değiştirilmeye dayanıklı hale getirilerek, sistemde aktif haldeki tüm cihazlara gönderilir ve eş zamanlı olarak saklanır. Burada işlenen bilgi, tüm cihazlara dağıtılacağı için gereksiz ve mükerrer bilgiden kurtulma amacıyla belirli bir hak ediş mekanizmasının çalıştırılması önemlidir. Bunun için Bitcoin (Nakamoto, 2008) blokzincirinde emeğin ispatı (proof-of-work) mekanizması çalıştırılır. Bu

\footnotetext{
* Sorumlu Yazar: Ford Otosan, Kurumsal Mimari ve Teknoloji İnovasyonu Departman1, İstanbul / Türkiye, ORCID: 0000-0002-5578-758X, mehmetaydar@gmail.com
} 
mekanizma ile sisteme eklenecek veri, kriptografik olarak güvence altına alınmakla birlikte, sistemi ayakta tutan madencilerin pasif durumdaki dijital paraları aktive etmesi ile sistemin sürdürülebilirliği sağlanır ve aynı zamanda sistemin güvenliği de arttırılmış olur.

Bu protokolde tüm işlemler, kullanıcılara ait açık ve gizli asimetrik anahtar çiftleri ile güvence altına alınır (Aydar et al., 2019). A ̆g üzerinde işlem yapmak isteyen kullanıcı, kendine ait olan ve sadece kendisi tarafından bilinmesi mümkün olan gizli anahtarı ile bu işlemi imzalar ve ağa yayınlar. Blokzincir protokolünün özgün bir şekilde ortaya koyduğu referanslı blok mekanizması, dağıtık ve kriptografik yapısı, bilgi saklamayı ve saklanan bilginin değiştirilemez hale getirilmesini kolaylaştırmaktadır. Geçmişe yönelik herhangi bir bilginin değiştirilmesinin neredeyse imkânsız hale getirilmesi, blokzincirin en güçlü yanlarınandan biri olarak kabul edilmektedir. Bunun yanında dağıtık yapısı ile tek merkeze yapılan ve sistemi sekteye uğratmayı amaçlayan saldırılara (single point of failure) karşı da oldukça güçlüdür.

Tüm bu yönleriyle birçok sektör, blokzincir teknolojilerine ilgi duymaktadır. Bu teknoloji hayatımıza finansal servisler ile girmesine rağmen son dönemde farklı endüstri aktörlerinin yoğun şekilde odak noktası olmuştur. Sunmuş olduğu güçlü yönleri sebebiyle bu teknoloji üzerine sayısal kimlik (Aydar and Ayvaz, 2019), sigortacılık, yapay zeka (Krittanawong et al., 2020), büyük veri güvenliği, noterlik işlemleri, emlak endüstrisi, otonom teknolojiler (Ayvaz and Cetin, 2019) gibi bir çok alanda yoğun şekilde çalışılmaktadır. Bu araştırmamızın konusu olan sağlık bilgi sistemlerinde de blokzincir protokollerinin önemli kullanım alanları mevcuttur.

\section{Materyal ve Metot}

\subsection{Sağlık Bilgi Sistemlerinde Kullanımı}

Sağlık sektörünün mevcut işleyişinde bazı alanlar, blokzincir protokolü aracılığıyla teknolojik dönüşüme oldukça uygundur ve büyük potansiyeller barındırmaktadır. Bu bölümde, blokzincir protokollerinin sağlık alanındaki mevcut kullanımı ve potansiyel kullanım alanları irdelenmiştir. Bunlar, sağlık bilgisi transferi, sağlık araştırmaları bütünlüğü, kişisel sağlık kayıtları, sağlık bilgisinin saklanması, faturalandırma, hasar kayıtları, ilaç tedarik zinciri ve pandemik durumlar şeklinde alt kullanım senaryolarına ayrılarak incelenmektedir.

\subsubsection{Să̆lık Bilgisi Transferi}

Blokzincir teknolojisi, bilgiye güvenli erişim sağlayan yapısı ile özellikle hastalar ve kurumlar arasındaki güvenli veri alışverişinin sağlanması konusunda büyük kolaylıklar sunmaktadır. Aralarında Mayo Clinic (Peterson et al., 2016), MIT (Azaria et al., 2016) ve hasta bilgisi erişimi üzerine çalışan kurumların da bulunduğu topluluklar tarafından, bu konsept üzerine çeşitli ürün makaleleri yayınlanmıştır. Bu modellerde genel olarak hasta bilgisi doğrudan blokzincir üzerine yüklenmez. Bunun yerine veri sahibi kurum veya veri gölleri (Linn and Koo, 2016) gibi harici bir noktadan referans verilir. Çin orijinli bir çalışma grubu, Healthcare Data Gateway isimli bir uygulama (Yue et al., 2016) ile hastaların blokzincir ağına bağlanarak kendi sağlık bilgilerini görüntüleyebilmesine imkan sunmuştur. Bu çalışmalar, genel olarak hasta öz kontrollü sağlık verisi konseptini desteklemektedir.

Healthcare Data Gateway projesi, hastaların kendi sağlık bilgisine kimin erişebileceğini kontrol edebilmesi üzerine kurulmuş bir konsept çalışmasıdır. Bu çalışma üç katmandan oluşmaktadır: (a) bilgi kullanım katmanı EMR (Elektronik Medikal Kayıt) sistemlerini, (b) veri saklama katmanı hasta bilgilerini doğrusal bir şema üzerinde tutan blokzincir bulut sistemini, (c) veri yönetimi katmanı ise akıllı cihazlar üzerinden hastalara kendi bilgilerine ulaşma ve yetkilendirme imkanı sağlayan uygulamayı bulundurmaktadir.

FHIRChain (Zhang et al., 2018), sağlık bilgisi üzerinde ortak çalışma (FHIR) prensibini, akıllı kontrat bazlı sağlık verisi paylaşımı açısından ele alarak geliştirilmiş bir blokzincir uygulamasıdır.

MedRec (Azaria et al., 2016) ise ethereum temelli bir blokzincir protokolü uygulamasıdır. Hastaların değiştirilemez sağlık verisi geçmişini yine hastaların onayları dâhilinde, farklı sağlık kuruluşlarıyla tedavi ve araştırma amaçlı paylaşımını hedeflemektedir. Proje üç ayrı akıllı kontrattan oluşmaktadır. Bunlar hastalar için kimlik doğrulayıcısı kontratlar, hasta kaydının saklanması işlemlerinden sorumlu kontratlar ve kayıtlar arası bağlantısal bilgilerden sorumlu kontratlardır.

MedChain (Shen et al., 2019), sağlık bilgisi sahiplerine, tedavi özelinde medikal bilgilerin paylaşılması yetkilerini vermek üzerine özelleşmiş, izne tabi katılımcılara hizmet veren bir uygulamadır.

Gem Health Network (Prisco, 2016) projesi de, ethereum tabanlı bir diğer blokzincir uygulamasıdır. Bu uygulama, paylaşımlı kimlik şemaları, veri saklama ve akıllı kontratlar üzerinden sağlık çalışanı uzmanlara en güncel sağlık verisini ulaştırmayı amaçlamaktadır.

MyPCR (MyP, 2018), yazılım güvenliği üzerine uzmanlaşmış Guardtime şirketi tarafindan desteklenen blokzincir destekli bir platformdur. 30 milyona varan hasta bilgisi ile Birleşik Krallık Sağlık Hizmetleri'ne hizmet vermesi planlanan proje, akıllı cihazlar üzerinden sağlık verisine anlık erişim sunmayı vaat etmektedir.

\subsubsection{Sağglık Araştırmaları Bütünlü̈̆̈̈̈}

Sağlık sektörü için teknolojik ilerlemenin temelini, fonlama sorunlarına rağmen akademik çalışmalar oluşturmaktadır. ClinicalTrials.gov ve benzeri organizasyonlar, bu alanda araştırma yapan bilim insanlarının problemlerini ortadan kaldırmayı ve çalışmaların önünü açmayı amaçlamaktadır. Blokzincir teknolojisi, sunduğu değiştirilemez, doğrulanabilir kayıtlar ve işlemler mekanizması ile biyomedikal araştırmacıları için dağıtık veri merkezi olarak kullanılabilmektedir. 2014 yılında, İngiltere'de birçok araştırmacı tarafından takip edilen Benjamin Carlisle (Carlisle, 2014), araştırmacıların projelerinde kullanabilmek üzere önceden belirlenmiş yönleri bulunan bilgiye blokzinciri üzerinden erişilebilineceğini ileri sürmüştür. Blokzincir, aynı zamanda bilgi bütünlüğü 


\section{Avrupa Bilim ve Teknoloji Dergisi}

sağladığı için, potansiyel araştıma verisinin kendisi halka açık olmasa dahi, doğruluğunu onaylamak için kullanılabilmektedir. Bu tip geliştirmeler, sağlık alanındaki araştırmalarının önünü açarken aynı zamanda medikal bilgilerin güvenliğini de sağlamaktadır.

Sync 4 Science, hastaların sayısal ortamda kayıtlı bulunan verilerinin bilimsel araştırmalarda kullanılması üzerine odaklanan bir pilot uygulamadir.

Healthbank (Dalianis et al., 2015), İsviçre temelli küresel bir sayısal sağlık girişimidir. Temelinde, hastaların sağlık verilerini blokzincir ağı üzerinde saklamaları ve bu veriyi kendi kontrolleri dahilinde sağlık araştırmacıları ile paylaşabilmeleri konsepti bulunmaktadır. Şirket, blokzincir protokolünden faydanlanarak hasta kayıtlarının sağlık araştırmaları kullanımındaki şeffaflığı arttırmayı amaçlamaktadır.

\subsubsection{Kişisel Să̆lık Kayıtları}

Bu konsept, blokzincir teknolojisinden faydalanarak, kişisel sağlık verisinin yüksek güvenlik çerçevesinde kontrol edilmesine odaklanmıştır. Bu alanda akademik ve konsept ispatı çalışmaları mevcuttur. eHealthWallet (Ehe, 2016), hastaların doktorları bilgi erişimi amacıyla yetkilendirebildiği bir konsept geliştirmiştir. Böylelikle hastalar, sağlık bilgilerini kendi kontrolleri dahilinde, tercih ettikleri doktorlarla paylaşabilmektedir.

Estonya merkezli eHealth projesi (Est, 2018), Guardtime firması ile ortak yürütülen, tedavi özelinde veri paylaşımına imkan sunan bir blokzincir projesidir.

Blokzincir bazlı HealthChain (Hylock and Zeng, 2019), hyperledger fabric ürünü üzerinde geliştirilmiş ve sağlık verisinin gizliliği ile Amerikan Sağlık Sigortası Taşınabilirlik ve Sorumluluk Yasası'na (HIPAA) uygunluk sağlamayı amaçlayan bir projedir.

MediBchain (Al Omar et al., 2017), izinli bir blokzincir protokolü kullanan ve veriye erişim için kimlik tanımlanması gerektiren bir mimaridir. Protokolde, kullanıcı bilgisinin asimetrik şifrelenmesine ek olarak, şifrelenmiş verinin bulunduğu bloğu ve blok numarasını saklar. Blok numarası, şifrelenmiş verinin blokzincir üzerinden çağırılması için kullanılır. Veri, kullanıcının gizli anahtarı ile deşifre edilir.

ModelChain (Kuo and Ohno-Machado, 2018), izinli bir blokzincir teknolojisi kullanan, her bir sağlık hizmeti sağlayıcısının kendi verilerini saklayabildiği ve gizlilik esaslı, makine öğrenmesiyle güçlendirilmiş bir blokzincir uygulamasıdır. Bu uygulama bilginin ispatı isimli yeni bir mutabakat mekanizmasını kullanmaktadır.

BlockchainHealth uygulaması, kişilerin sağlık verilerini takibi ve araştırmacılar ile paylaşması üzerine tasarlanmış blokzincir tabanlı bir portaldır. Bilgiye erişim ve paylaşım, değiştirilemez geçmiş prensibiyle kayıt altına alınmakta ve resmi yetkililer tarafından aynı anda takip edilebilmektedir.

eHealthWallet (Ehe, 2016) uygulaması, ethereum blokzinciri üzerinde geliştirilmiş, sağlık verisi saklama ve paylaşma platfomudur.

2015 yılında konsept ispatı çalışmaları paylaşılan MedVault (Blough et al., 2011) ürününde, hasta verisi bitcoin ağında tutulmaktadır. Hastalar, kendilerine verilen karekod ile ağ üzerinde oturum açabilmekte, doktorlar da hastaların kayıtlarına aynı şekilde erişebilmektedir.

\subsubsection{Să̆lık Bilgisinin Saklanması}

Bu bölüme kadar verilen örneklerin çoğu, blokzincir protokolünü doğrudan veri saklama amacıyla değil güvenli referans gösterim noktası olarak kullanmayı tercih eden uygulamalardır. Philips Blokzincir Labratuvarı ortaklarından olan Tierion isimli şirketten bir grup, hasta bilgilerini blokzincir üzerinde saklamayı baz alan, merkle ağacı ve mevcudiyetin ispatı konseptlerini kullanan Chainpoint (Vaughan et al., 2016) isimli bir ürün ortaya koymuşlardır. Benzer şekilde, MedVault (Blough et al., 2011) adlı çalışmada, hasta sağlık bilgisini doğrudan blokzincir üzerinde saklamak için Colu blokzincir protokolü tercih edilmiştir.

\subsubsection{Faturalandırma ve Hasar Kayttlart}

Blokzincir teknolojisi, akıllı sözleşmeler ile, bilgi bildirimi ve ödeme sistemlerinde yönetim maliyetlerini düşürmesinin yanında, zaman tasarrufu sağlamıştır. Bunun sağlık sektöründeki yansıması, ödemeyi yapacak taraf -bu bir kişi ya da bir kurum olabilir- ile hasta ve sağlık hizmetini verecek kurum arasında, hizmet faydalandırımı gerçekleştikten sonra ödemenin otomatik olarak tamamlanması üzerine düzenlenmiş bir akıllı kontrat olabilir. Burada normal prosedür işleyişinin aksine, sigorta şirketinin belgelerin incelemesi, tasdiklemesi, ödeme onayının verilmesi ve ödemenin gerçekleşmesi aşamaları güvenle tamamlanmış ve zaman tasarrufu sağlanmış olur (Srinivasan, 2017).

\subsection{6. İlaç Tedarik Zinciri ve Reçete Yönetimi}

Özellikle geri kalmış ve gelişmekte olan ülkelerde sahte ilaç oranı neredeyse 1/10'dur ve bu ülkelerde sahte ilaçlar, sağlık sektöründeki büyük problemlerden biridir (WHO, 2017). Hasta güvenliğinin teminatı, sağlık sektörü için kritik ve öncelikli konulardan biridir. Bu yüzden ilaçların üretiminden tüketimine kadar olan sürecin doğruluğu, bütünlüğü, takip edilebilirliği ve güvenliği elzemdir. Bu konuda blokzincir protokolleri ve akıllı kontratlar, ilaçların ve reçetelerin yönetiminde, geçmişinin ve gerçekliğinin takip edilmesinde kullanılabilir (Bocek et al., 2017).

\subsubsection{Pandemi ile Mücadele}

Pandemik hastalıklar, hızlı yayılmaları durumunda sağlık sistemi üzerindeki yükü oldukça arttırmakta ve sektörü kötü niyetli faaliyetlere karşı savunmasız duruma düşürebilmektedir. Örneğin, Dünya Sağlık Örgütü (WHO) tarafından 11 mart 2020 tarihinde 
pandemi ilan edilen yeni tip coronavirüsü (COVID-19) (WHO, 2020), oldukça hızlı yayılım göstermesinden dolayı birçok ülkede sağlık sistemini zayıflatmıştır.

Amerikan yayın organı The New York Times 16 mart 2020 tarihli yazısında (Sanger et al., 2020), COVID-19 sebebiyle oluşan panik esnasında kötü niyetli kişilerin sağlık sektöründe hassasiyetle korunması gereken bazı kayıtlara yaptığı saldırıları ele almaktadır. The New York Times siber güvenlik raportörü aynı zamanda twitter üzerinden yaptığı paylaşımlarda, doktorlardan gelen çağrıya değinmiş ve Silikon Vadisi'ndeki teknoloji devlerinin COVID-19 ile mücadeleye yardımcı olmak için geliştirmesi gereken uygulamalara değinmiştir (twi a 2020, twi b 2020). İrdelenen konu özelinde, doktorların COVID-19 ve enfekte olan hastalara ait bilgileri izinli bir platformda paylaşabileceği ve bilginin güvenilir sağlı personelleri tarafindan paylaşıldığına ve değiştirilmediğine emin olacakları bir platform öne çıkmaktadır. İhtiyaç duyulan bu platform, Sovrin (Tobin and Reed, 2016) ve Uport (Lundkvist et al., 2017) gibi blokzincir temelli öz-egemen kimlik sistemleri ile mümkün olabilmektedir. Bu senaryoda, sağlık çalışanlarına blokzincir üzerinden asimetrik anahtarlar kullanılarak kimlik atanır. Çalışanlar paylaşmak istedikleri veriyi, özel anahtarlarıyla imzalayarak verinin doğrulanabilir referansını (Sporny et al., 2019) oluştururlar ve ilgili platform üzerinden paylaşırlar. Platform üzerinden, veriyi paylaşanın açık anahtarı yardımıyla ilgili verinin orijinalliği ve değiştirilmediği kolaylıkla teyit edilebilir.

MiPasa (mipasa, 2020) adı verilen blokzincir tabanlı proje, COVID-19 taşıyıcılarını erken teşhis etmek amacı ile kişiler, devlet kurumları ve sağlık kuruluşlarının mahremiyetini koruyarak birbirleriyle veri paylaşımına olanak sağlar. Hyperledger Fabric blokzincir teknolojisini kullanan MiPasa üzerinde, teyit edilmiş veri üzerinden analiz yapmak için yapay zeka algoritmaları çalıştırılabilir. MiPasa, aralarında IBM, Microsoft, Dünya Sağlık Örgütü, John Hopkins Üniversitesi ve Çin Milli Sağlık Komisyonu gibi kuruluşlardan oluşan bir konsorsiyum tarafından desteklenmektedir.

Doğrudan pandemi ile ilgili olmasa da, blokzincirin kullanım senaryoları arasında bulunan bağış takibi, sigorta tazminat talebi, medikal ekipman tedarik zincirinin takip edilmesi, salgın durumlarında verinin izlenmesi ve uluslararası ödemeler, pandemik durumlarda birer ihtiyaç olarak ön plana çıkmaktadır.

\section{Araştırma Sonuçları ve Tartışma}

Blokzincir protokolleri, daha önce de bahsedildiği gibi, üzerinde barındırdığı güçlü kriptografi algoritmaları, konsensüs mekanizması ve dağıtık mimarisi ile güven makinası olarak düşünülebilir. Aynı zamanda hayatımıza girişi itibariyle bir ödeme ve değer transferi aracı olarak da ele alınabilmektedir. Özellikle üzerinde çalışmaya imkan sunduğu akıllı kontratlarla birlikte, işleri otomatize eden bir yapıya dönüştürülebilmektedir.

Sağlık bilgi sistemlerinde blokzincir kullanımının salt faydalarından bahsetmek gerçekçi olmayacaktır. Öncelikle temel faydalarının daha anlaşılabilir olması için, blokzincir teknolojisinin ortaya atılırken amaçladığı sorunların çözümüne bakılması daha doğrudur. Örneğin blokzincir vasıtasıyla, işlemin tarafları arasındaki üçüncü parti kuruluşlar ortadan kaldırılabilmektedir. Bir diğer avantajı, taraflar arasında şeffaf işlem kabiliyeti sağlanması ve bu şeffaflığın yüksek güvenlik ve taraflara ait mahremiyet dahilinde sağlanmasıdır. Aynı zamanda, işlem geçmişinin dağıtık olarak saklanması, veri saklama ve sunucu hizmeti sağlayan kurumların tekelinden kurtulunması ve belirli noktalara yapılan ve sistemin çalışmasını sekteye uğratan saldırılara karşı ek güvenlik sunması, blokzincirin avantajları arasında değerlendirilebilir. Ek olarak, blokzincir uygulamaları genellikle açık kaynak kodludur ve hiçbir kurumun tekelinde değildir. Böylelikle çeşitli topluluklar tarafından desteklenmekte ve her geçen gün daha iyiye götürülmeye çalışılmaktadır.

Birden fazla protokolün aynı anda farklı projelerde desteklenmesi ihtiyacı ve bu protokoller arasındaki entegrasyon sorunları, blokzincir teknolojisinin zayıf yönleri arasında gösterilebilir. Ayrıca dağıtık yapısı yüzünden güçlü depolama alanlarına ihtiyaç duyulabilmektedir. Güvenliğin getirdiği kompleks mimarisi, yer yer değişiklik gösterse de kullanılan mutabakat mekanizmasına göre yüksek enerji tüketimi, test edilebilirlik ve son kullanıcıya ulaştırılması konusundaki sorunlar da dezavantajlı yönleri olarak ele alınabilir. Bu bağlamda, blokzincir teknolojisinin güçlü ve zayıf yönleri Tablo 1 de özetlenmiştir.

Tablo 1: Blokzincir teknolojisinin güçlü ve zaylf yönleri

\begin{tabular}{|l|l|}
\hline Güçlü Yönler & Zayıf Yönler \\
\hline Şeffaflık ve geçmişe dönük değişikliğe direnç & Farklı protokollerin senkron çalışamaması \\
\hline Dağıtık mimari & Son kullanıcıya uzaklık \\
\hline Hızlı işlem imkanı & Karmaşık mimari, düşük test edilebilirlik \\
\hline Açık kaynak kodu & Yüksek beceri gerekliliği \\
\hline Kriptografi sayesinde yüksek güvenlik & Henüz olgunlaşmamış olması \\
\hline Sorgulanamaz doğruluk kabulü & Yeni teknolojinin kabulü zorlukları \\
\hline
\end{tabular}


chinemoneynetwork.com sitesinin paylaştı̆̆g 2018 mayıs tarihli ve Çin Bilgi ve İletişim Teknolojileri Akademisi'nden (CAICT) bir yetkiliye dayandırdığ haberde, 80.000 blokzincir girişiminden sadece $\% 8$ inin hayatına devam edebildiği ve bu girişimlerin ortalama ömrünün 1.22 sene olduğu belirtilmiştir (China Money AI, 2018). Aynı yetkili, blokzincir projelerinin çok hızlı yükselmesine karşılık başarısızlıkla erken tanıştığını belirtmiştir. Forbes’ta yayınlanan 2019 mayıs tarihli makalede ise (Disparte., 2019) blokzincir projelerinin başarısızlığının temel sebebi, girişimlerin temellerinin atıldığı sektörlerin dijital dönüşüme henüz hazır olmaması ve dolayısıyla gerekli fizibilite ve altyapı çalışması eksikliği olarak gösterilmektedir. Fakat sağlık sektöründe, yıllardır süregelen dijitalleşme ve dönüşüme ek olarak blokzincir uygulamaları entegrasyonunun uygunluğu aşikardır.

Tüm bu bilgiler 1şı̆̆ında blokzincir protokollerinin, dezavantajları bulunmasına rağmen, hassas sağlık verisinin mahremiyet çerçevesinde güvenle korunması, paylaşımı, işlenmesi için oldukça avantajlı olduğu açıkça görülmektedir. Sağlık sektörünün mahremiyet ve güvene dayalı bilgi kaydı ve transferi ihtiyacıyla, blokzincir protokollerinin doğasından gelen güçlü yanları eşleştiğinde ortaya somut örnekler çıkmaya başlamıştır. Belirtilen perspektifin efektif sonuçlarından biri, IBM tarafından özellikle COVID-19 pandemisi döneminde hızla hayata geçirilerek sağlık sektörünün hizmetine sunulan IBM Rapid Supplier Connect ürünüdür (Kelley, 2020). Bu ürün sayesinde, ticari kaygılar gütmeyen aracı kuruluşları da içerisine alan alıcı ve tedarikçiler, sağlık sektöründe kullanılan ürünlerin tedariği için kolaylıkla iletişim kurabilmektedir. Yine IBM, Walmart, Merck ve KPMG’nin ortak yayınladığı Blokzincir Birlikte Çalışılabilirlik Pilot Projesi raporunda, çalışmamızda değindiğimiz konuları da içine alan araştırmaların sonucu olarak blokzincir ürünlerinin sağlık alanındaki bazı uygulamalarının efektif sonuçları test edilmiş ve paylaşılmıştır (FDA, 2020). Birleşik devletler gıda ve ilaç yönetimi örgütü için hazırlanan bu rapora göre, blokzincir teknolojisi ilaç tedariği ve ilaçların geri çağrılması durumlarında takip edilebilirlik ve hızlı aksiyon amacıyla kullanılabilmektedir. Raporda önerilen iki yaklaşım, ilaç tedarik akışının izlenmesi ve uyarı sistemlerinin aktif ve süratli bir şekilde hayata geçirilmesidir. Yine bu raporda ele alınan sonuçlar incelendiğinde, pilot çalışmaların iki yaklaşımı da başarıyla hayata geçirebileceği görülmektedir.

\section{Sonuç}

$\mathrm{Bu}$ araştırmada, blokzincir teknolojisinin sağlık bilgi sistemleri özelinde, temel teknik çalışma prensipleri, avantajları ve dezavantajları ile genel kullanım alanları irdelenmiştir. Sağlık sistemlerinde blokzincir kullanımında tek bir blokzincir modeli ya da kullanım senaryosundan bahsetmek mümkün değildir. Çalışmamızda aynı zamanda blokzincirin sağlık sektörüne sunabileceği potansiyel faydalar ve hali hazırda geliştirilmiş veya geliştirilmekte olan blokzincir tabanlı ürünler ile kavram ispatları ele alınmıştır. Sağlık alanındaki mevcut blokzincir çalışmaları, sağlık bilgisi değişimi, sağlık araştırmaları bütünlüğü, kişisel sağlık kayıtları, sağlık bilgisinin saklanması, faturalandırma, hasar kayıtları ve ilaç tedarik zinciri ve pandemik durumlarda kullanımı olarak alt kullanım senaryolarında ele alınmıştır. Çalışmamız, blokzincir teknolojisinin sağlık alanında kullanımı ile ilgili araştırmalara ışık tutacaktır.

\section{Kaynakça}

[Vaughan et al., 2016] (2016) Vaughan W., Bukowski J., Wilkinson S. Chainpoint: A scalable protocol for anchoring data in the blockchain and generating blockchain receipts.

[Sanger et al., 2020] (2020) Sanger D. E., Perlroth N., Rosenberg M. Hackers attack health and human services computer system.https://www.nytimes.com/2020/03/16/us/politics/coronavirus-cyber.html.Accessed: 2020-03-20.

[Srinivasan, 2017] (2017) Srinivasan P. Healthcare Blockchain: How Smart Contracts Could Revolutionize CareDelivery. [mipasa, 2020] (2020) MiPasa. https://mipasa.org/.

[twi, 2020] 2020 (2020 Nyt cybersecurity $\quad$ reporter's reply. https://twitter.com/nicoleperlroth/status/1239723703706869761?s=20.Accessed: $2020-03-20$.

$\left[\begin{array}{lllll}\text { twi, } & \text { b } & 2020] & \text { (2020) } & \text { Nyt }\end{array}\right.$ https://twitter.com/nicoleperlroth/status/1239723379151650821?s=20.Accessed: 2020-03-20.

[WHO, 2020] (2020) Who director-general's opening remarks at the media briefingon covid-19. https://www.who.int/dg/speeches/detail/who-director-general-s-opening-remarks-at-the-media-briefing-on-covid-19-11-march2020.Ac-cessed: 2020-03-20.

[Carlisle, 2014] (2014). Carlisle, B. G. Proof of prespecified endpoints in medical research with the bitcoin blockchain.http://blog.bgcarlisle.com/2014/08/25/proof-of-prespecified-endpoints-in-medical-research-with-the-bitcoinblockchain/.[Online; accessed 20-Jan-2020].

[Ehe, 2016] (2016). Healthy SELFIE e-Health Wallet. https://devpost.com/software/ehealthwallet.[Online; accessed 20-Jan-2020].

[WHO, 2017] (2017). 1 in 10 medical products in developing countries is substan-dard or falsified. https://www.who.int/en/newsroom/detail/28-11-2017-1-in-10-medical-products-in-developing-countries-is-substandard-or-falsified.[Online; accessed 20-Jan2020].

[Est, 2018] (2018).Estonian eHealth Authority Partners with Guard-time to Accelerate Transparency and Auditability in Health Care.https://guardtime.com/blog/estonian-ehealth-partners-guardtime-blockchain-based-transparency. [Online; accessed 20-Jan-2020].

[MyP, 2018] (2018).World's first blockchain-supported Personal CareRecord Platform launched by Guardtime and partners to up to 30 millionNHS patients in the UK .https://guardtime.com/blog/world-s-first-blockchain-supported-personalcare-record-platform-launched-by-guardtime-and-partners. [Online; accessed 20-Jan-2020].

[Al Omar et al., 2017] Al Omar, A., Rahman, M. S., Basu, A., and Kiy-omoto, S. (2017). Medibchain: A blockchain based privacy preserving plat-form for healthcare data. InInternational conference on security, privacyand anonymity in computation, communication and storage, pages 534-543.Springer. 
[Aydar and Ayvaz, 2019] Aydar, M. and Ayvaz, S. (2019).Towards ablockchain based digital identity verification, record attestation and recordsharing system.arXiv preprint arXiv:1906.09791.

[Aydar et al., 2019] Aydar, M., Cetin, S. C., Ayvaz, S., and Aygun, B. (2019).Private key encryption and recovery in blockchain.

[Ayvaz and Cetin, 2019] Ayvaz, S. and Cetin, S. C. (2019). Witness of things.International Journal of Intelligent Unmanned Systems.

[Azaria et al., 2016] Azaria, A., Ekblaw, A., Vieira, T., and Lippman, A.(2016). Medrec: Using blockchain for medical data access and permissionmanagement. In2016 2nd International Conference on Open and Big Data(OBD), pages 25-30. IEEE.

[Blough et al., 2011] Blough, D. M., Liu, L., Sainfort, F., and Ahamad, M.(2011). Ct-t: Medvault-ensuring security and privacy for electronic medicalrecords. Technical report, Georgia Institute of Technology.

[Bocek et al., 2017] Bocek, T., Rodrigues, B. B., Strasser, T., and Stiller, B.(2017). Blockchains everywhere-a use-case of blockchains in the pharmasupply-chain. In2017 IFIP/IEEE symposium on integrated network and ser-vice management (IM), pages 772-777. IEEE.

[Dalianis et al., 2015] Dalianis, H., Henriksson, A., Kvist, M., Velupillai, S., andWeegar, R. (2015). Health bank-a workbench for data science applications inhealthcare. InCAiSE Industry Track, pages 1-18.

[Hylock and Zeng, 2019] Hylock, R. H. and Zeng, X. (2019). A blockchainframework for patient-centered health records and exchange (healthchain):Evaluation and proof-of-concept study.Journal of medical Internet research,21(8):e13592.

[Krittanawong et al., 2020] Krittanawong, C., Rogers, A. J., Aydar, M., Choi,E., Johnson, K. W., Wang, Z., and Narayan, S. M. (2020). Integratingblockchain technology with artificial intelligence for cardiovascular medicine.Nature Reviews Cardiology, 17(1):1-3.8

[Kuo and Ohno-Machado, 2018] Kuo, T.-T. and Ohno-Machado, L. (2018).Modelchain: Decentralized privacy-preserving healthcare predictive modelingframework on private blockchain networks.arXiv preprint arXiv:1802.01746.

[Linn and Koo, 2016] Linn, L. A. and Koo, M. B. (2016). Blockchain for healthdata and its potential use in health it and health care related research.InONC/NIST Use of Blockchain for Healthcare and Research Workshop.Gaithersburg, Maryland, United States: ONC/NIST, pages 1-10.

[Lundkvist et al., 2017] Lundkvist, C., Heck, R., Torstensson, J., Mitton, Z., and Sena, M. (2017). Uport: A platform for self-sovereign identity.URL:https://whitepaper. uport. me/uPortwhitepaperDRAFT20170221. pdf.

[Nakamoto, 2008] Nakamoto, S. (2008). Bitcoin: A peer-to-peer electronic cashsystem. Technical report, Manubot.

[Peterson et al., 2016] Peterson, K., Deeduvanu, R., Kanjamala, P., and Boles,K. (2016). A blockchain-based approach to health information exchange net-works. InProc. NIST Workshop Blockchain Healthcare, volume 1, pages 1-10.

[Prisco, 2016] Prisco, G. (2016). The blockchain for healthcare: Gem launchesgem health network with philips blockchain lab.Bitcoin Magazine, 26.

[Shen et al., 2019] Shen, B., Guo, J., and Yang, Y. (2019). Medchain: efficienthealthcare data sharing via blockchain.Applied sciences, 9(6):1207.

[Sporny et al., 2019] Sporny, M., Longley, D., and Chadwick, D. (2019). Ver-ifiable credentials data model 1.0.W3C, W3C Candidate Recommendation,March.

[Tobin and Reed, 2016] Tobin, A. and Reed, D. (2016). The inevitable rise ofself-sovereign identity.The Sovrin Foundation, 29(2016).

[Yue et al., 2016] Yue, X., Wang, H., Jin, D., Li, M., and Jiang, W. (2016).Healthcare data gateways: found healthcare intelligence on blockchain withnovel privacy risk control.Journal of medical systems, 40(10):218.

[Zhang et al., 2018] Zhang, P., White, J., Schmidt, D. C., Lenz, G., and Rosen-bloom, S. T. (2018). Fhirchain: applying blockchain to securely and scal-ably share clinical data.Computational and structural biotechnology journal,16:267-278.

[China Money AI, 2018] (2018). Chinese Official Says Blockchain Projects Globally Had Average Life Span Of 1.22 Years. https://www.chinamoneynetwork.com/2018/05/28/chinese-official-says-blockchain-projects-globally-had-average-life-span-of-122-years. [Online; accessed 20-May-2020].

[Disparte., 2019] Disparte D. A. Why Enterprise Blockchain Projects Fail. Forbes.

[Kelley, 2020] (2020) Kelley J. IBM Rapid Supplier Connect: Getting COVID-19 responders tehe equipment they need.

[FDA, 2020] (2020) Blockchain Interoperability Pilot Project Report. 